\title{
Will cosmic acceleration last forever?
}

\author{
J. S. Alcaniz \\ Observatório Nacional, Rio de Janeiro - RJ, 20921-400, Brasil \\ email: alcaniz@on.br
}

Although the transition from an initially decelerated to a late-time accelerating cosmic expansion is becoming observationally established, the duration of the accelerating phase depends on the cosmological scenario and, several models, which includes our standard one, imply an eternal acceleration or even an accelerating expansion until the onset of a future cosmic singularity. In this regard, an interesting theoretical question arises if one tries to reconcile the standard description of the current cosmic acceleration with the only candidate for a consistent quantum theory of gravity we have today, i.e., Superstring theory.

As is well known, in the standard cosmological scenario, after radiation and matter dominance, the Universe asymptotically enters a de Sitter phase with the scale factor $a(t)$ growing exponentially, which results in an eternal cosmic acceleration. In such a background, the cosmological event horizon

$$
\Delta=\int_{t_{0}}^{\infty} \frac{d t}{a(t)} \rightarrow \text { converges }
$$

and this is particularly troublesome for the formulation of String/M theory because local observers inside their horizon are not able to isolate particles to be scattered, which implies that a conventional S-matrix cannot be built (see e.g. Fischler et al. (2001), Hellerman et al. (2001)). This dark energy/String theory conflict, therefore, leaves us with the formidable task of either finding alternatives to the conventional S-matrix or constructing a model for the Universe that predicts the possibility of a transient acceleration phenomenon.

In fact, this latter possibility can be achieved in the context of the so-called thawing scalar field models in which a new deceleration period will take place in the futuret. Examples of transient cosmic acceleration can also be found in brane-world cosmologies (Sahni and Shtanov (2003)), as well as in models of coupled quintessence (interacting dark matter/dark energy), as recently discussed by Costa and Alcaniz (2009).

Another interesting example of transient cosmic acceleration was recently discussed by Alcaniz et al. (2009). In this scenario, the field potential provides an equation-of-state (EoS) parameter of the type

$$
w(a)=-1+\text { const. }\left(a^{\kappa}+a^{-\kappa}\right)^{2},
$$

in which the competition between the double scale factor terms gives rise to a hybrid behavior: it is freezing over all the past cosmic evolution, is approaching the value -1 today (in agreement with current obsevational limits on $w$ ), will become thawing in the near future and will behave as such over the entire future evolution of the Universe. This behavior clearly leads to a transient acceleration phase, as shown in Fig. 1. Note that

$\dagger$ Thawing models describe a scalar field whose the equation-of-state parameter increases from $w \sim-1$, as it rolls down toward the minimum of its potential, whereas freezing scenarios describe an initially $w>-1$ EoS decreasing to more negative values (Caldwell and Linder (2005)) 


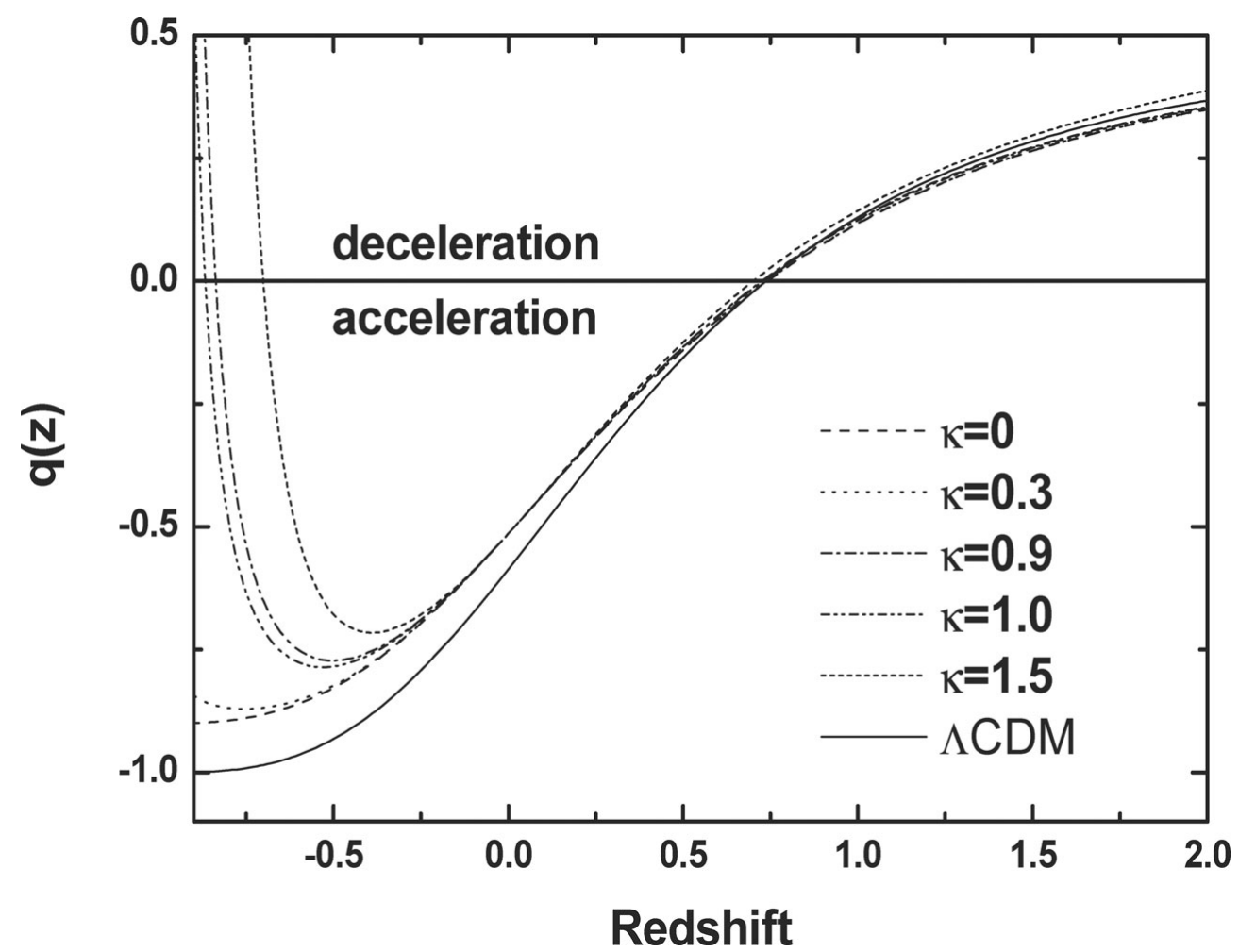

Figure 1. Cosmic deceleration/acceleration history for some selected values of $\kappa$. For comparison, the $\Lambda \mathrm{CDM}$ evolution is also shown.

similarly to thawing models, for hybrid scenarios the cosmological event horizon

$$
\Delta=\int_{t_{0}}^{\infty} \frac{d t}{a(t)} \rightarrow \text { diverges }
$$

thereby allowing the construction of a conventional S-matrix describing particle interactions within the String/M-theory frameworks.

Finally, an important aspect worth emphasizing at this point is that, although thawing and hybrid models may provide a possible way to reconcile the observed acceleration of the Universe with theoretical constraints from String/M theories, they are distinguishable in what concerns the past cosmic evolution. In this regard, some recent analyses using current data from SNe Ia, LSS and CMB have explored possible variations in the $w-a$ plane and indicated a slight preference for a freezing behavior over the thawing one (Krauss et al. (2007), Zunckel and Trotta (2007)). For instance, Huterer and Peiris (2007) uses the Monte Carlo reconstruction formalism to scan a wide range of possibilities for $w(a)$ and find that $\sim 74 \%$ are for freezing whereas only $\sim 0.05 \%$ are for thawing. Similar conclusions are also obtained by Zunckel and Trotta (2007) by using the socalled maximum entropy method, where the HST/GOODS SNe Ia data showed $\simeq 1 \sigma$ level preference for $w>-1$ at $z \sim 0.5$ with a drift towards $w>-1$ at higher redshifts. If such a preference for freezing EoS persists even after a systematically more homogeneous and statistically more powerful data sets become available, the combination of theoretical and observational constraints may give rise to an interesting case for hybrid models. 


\section{References}

W. Fischler, A. Kashani-Poor, R. McNees, \& S. Paban, JHEP 3, 0107 (2001)

S. Hellerman, N. Kaloper, \& L. Susskind, JHEP 3, 0106, (2001)

R. R. Caldwell \& E. V. Linder, Phys. Rev. Lett. 95, 141301 (2005).

F. C. Carvalho, J. S. Alcaniz, J. A. S. Lima, \& R. Silva, Phys. Rev. Lett. 97, 081301 (2006).

J. S. Alcaniz, F. C. Carvalho, Z.-H. Zhu, \& R. Silva, Class. Quantum Grav. 26105023 (2009).

V. Sahni \& Y. Shtanov, JCAP 0311, 014 (2003).

F. E. M. Costa \& J. S. Alcaniz, Phys. Rev. D 81, 043506 (2010).

L. M. Krauss, K. Jones-Smith, \& D. Huterer, New J. Phys. 9, 141 (2007).

C. Zunckel \& R. Trotta, Mon. Not. Roy. Astron. Soc. 380, 865 (2007).

D. Huterer \& H. V. Peiris, Phys. Rev. D 75, 083503 (2007). 